\section{Radiotherapy alone is suitable in older patients with early- stage Hodgkin lymphoma}

Adding chemotherapy to radiotherapy regimens can increase response and relapse-free survival in patients with Hodgkin lymphoma $(\mathrm{HL})$ who have an unfavorable prognosis; however, the poorer prognosis of older patients and their decreased tolerance of chemotherapy means that this group is usually initially treated with radiotherapy alone. Landgren et al. performed a long-term population-based cohort study in consecutive patients with early-stage $\mathrm{HL}$ treated initially with radiotherapy alone, and compared relapse rate, outcome and predictors of prognosis between older and younger patients.

Following radiotherapy, 218 (90\%) of patients aged $<60$ years and $54(82 \%)$ of those aged $\geq 60$ years achieved complete remission. Relapse rates were similar between the two age-groups ( $42 \%$ in total), and neither gender nor age at diagnosis were associated with risk of relapse. The prognosis of older patients who relapsed and received rescue chemotherapy was similar to that shown in other studies for older patients treated initially with chemotherapy. There was no acute radiotherapy-related mortality, and the sites of secondary neoplasms were unrelated to applied radiation field in older patients, suggesting that radiotherapy was not the cause.

The authors conclude that there is no evidence of an age-related risk of relapse in older patients with early-stage $\mathrm{HL}$ treated with radiotherapy alone, and that this could be a suitable alternative first-line therapy in this group. They believe that the decision to use chemotherapy in older patients should be based on individual risk profile, projected benefit versus risk of iatrogenic morbidity/mortality, and consultation with the patient.

Original article Landgren $\mathrm{O}$ et al. (2006) A populationbased cohort study on early-stage Hodgkin lymphoma treated with radiotherapy alone: with special reference to older patients. Ann Oncol 17: 1290-1295

\section{Gene expression profiles for renal cell carcinoma and renal regeneration show similarity}

It has been suggested that cancers are analogous to wounds that do not heal. To examine whether cancer and tissue regeneration share common molecular phenotypes, Riss et al. used a microarray approach to compare the gene expression profiles underlying human renal cell carcinoma $(\mathrm{RCC})$ and renal regeneration and repair (RRR).

The researchers compared the gene expression profile of RRR from a mouse model of unilateral renal ischemia and reperfusion with that of a set of genes differentially expressed in RCC relative to normal kidney samples identified through a literature search. They identified 361 genes that were differentially expressed in both $\mathrm{RRR}$ and RCC. Of the differentially expressed genes, $77 \%$ were concordantly regulated, revealing a significant similarity between the two gene expression signatures $\left(P=2.2 \times 10^{-16}\right)$ and supporting the concept of cancers as wounds that do not heal. The remaining 83 genes were discordantly expressed in RRR and RRC (i.e. upregulated genes in RCC were downregulated in RRR and vice versa). Pathway and gene ontology analysis of the concordant gene group revealed that normal RRR processes such as cell proliferation and growth, translation, immune response and metabolism also occur in RCC. By contrast, the discordant gene list was enriched in genes involved in morphogenesis, glycolysis, and the hypoxia-inducible factor and insulinlike growth factor 1 pathways, which might reflect RCC pathogenesis. Further analysis of the discordantly regulated genes might provide insight into the differences between normal tissue regeneration and malignancy and may help develop novel diagnostic and therapeutic targets specific to carcinoma.

Original article Riss J et al. (2006) Cancers as wounds that do not heal: differences and similarities between renal regeneration/repair and renal cell carcinoma. Cancer Res 66: 7216-7224

\section{Anastrozole preferred to tamoxifen for treatment of early-stage breast cancer}

The Arimidex, Tamoxifen, Alone or in Combination (ATAC) trial concluded that anastrozole was more effective than tamoxifen in preventing recurrence of breast cancer. The ATAC Trialists' Group recently reported results from a detailed analysis of the safety and tolerability of the two compounds and combined these data with efficacy outcomes to produce overall risk-benefit indices. The researchers found 